\title{
Coherent Odds and Subjective Probability
}

\author{
Kim C. Border* Uzi Segal ${ }^{\dagger}$
}

October 11, 2001

\section{Introduction}

Expected utility theory, first axiomatized by von Neumann and Morgenstern [33], was considered for many years to be the only acceptable normative theory for decision making under risk. von Neumann and Morgenstern assumed the existence of probabilities, but it was soon proved that this assumption is unnecessary, and the theory is meaningful even with subjective probabilities (see Savage [29] and Edwards [8]). Despite the experimental criticism of this theory (e.g. Allais [2] or MacCrimmon and Larsson [21]), it was not until the late 1970 's that serious alternatives to this theory were offered (e.g. Kahneman and Tversky [17] or Machina [22]). The most popular of these alternatives, called the rank-dependent utility model (Quiggin [27]; see also further references in Section 2 below), assumes that decision makers maximize an expected utility-type functional, where instead of the original distribution function they use a (subjective) transformation of this function. Another interpretation of this idea suggests that decision maker use nonadditive probabilities (Schmeidler [30]. For some recent applications and axiomatizations of these theories and their extensions, see Abdellaoui [1], Lopes and Oden [18], Miyamoto [25], Luce [19], Tversky and Kahneman [32], or Wakker and Tversky [35]).

One of the strongest arguments against using non expected utility models is that they lead to Dutch books, that is, to situations where a decision maker

\footnotetext{
*Division of the Humanities and Social Sciences, California Institute of Technology, Pasadena CA 91125, USA

${ }^{\dagger}$ Department of Economics, Boston College, Chestnut Hill MA 02467, USA
} 
can be manipulated to lose money with probability one (see Machina [24]). In the context of non-additive probabilities, this claim is based on the following argument by de Finetti [7].

A set of odds posted by a bookie is coherent if it is impossible to make a sure profit by betting against the bookie. de Finetti [7, p. 63], among others, has argued that

[it is] precisely this condition of coherence which constitutes the sole principle from which one can deduce the whole calculus of probability: this calculus then appears as a set of rules to which the subjective evaluation of probability of various events by the same individual ought to conform if there is not to be a fundamental contradiction among them.

The basis for this claim is the following theorem due originally to de Finetti. A set of odds is coherent if and only if they are derived from a finitely additive probability measure. (A very general version of this result may be found in Heath and Sudderth [16]). To illustrate, assume that a bookie posts odds of $\frac{1}{3}$ on $A, \frac{1}{3}$ on $B$, but $\frac{1}{2}$ on $A \cup B$, even though $A \cap B=\varnothing$. Then a smart bettor will sell the bookie a bet that pays $\$ 1$ if $A$ happens, charging her $\frac{1}{3}-\varepsilon$, he will sell her another bet that pays $\$ 1$ if $B$ happens for the same price, and pay her $\frac{1}{2}+\varepsilon$ for a bet that pays $\$ 1$ is $A \cup B$ happens. For $\varepsilon<\frac{1}{18}$, the bettor ends up with a sure gain of $\frac{1}{6}-3 \varepsilon>0$.

de Finetti's argument is normative. It does not necessarily apply to actual behavior, but is more about an individual's beliefs. Such beliefs are normatively acceptable if the individual knows that it is impossible to exploit money out of her, and de Finetti shows that this requirement is satisfied by posting coherent odds, that is, odds that behave like a probability function. We do not wish to argue with the normative appeal of de Finetti's argument, but would like to ask another question: Is it true that a bookie with incoherent odds will be exploited, in the sense that she will necessarily lose money? As mentioned above, this claim is often cited against some of the current models used by psychologists and economists, where non-additive representations of beliefs are sometimes employed. The question whether such preferences must lead to bankruptcy is a practical, rather than a normative question. As we show below, when bookies act strategically, it may well be optimal for them to post incoherent odds. There is no contradiction between definition's analysis and our results. de Finneti is seeking immunization against all possible 
bettors, while we deal with a situation where the bookie interacts with a given set of bettors.

Consider again the requirement 'a set of rules to which the subjective evaluation of probability ... ought to conform,' not as a normative rule (with which we agree), but as a practical one. There are two behavioral interpretations we can make of this statement. One is that being in a betting environment forces a bookie to post odds in a way that makes her appear to have a subjective probability. The other is that she has a subjective probability and that placing her in a betting environment enables us to uncover her beliefs.

This second interpretation is clearly flawed. In a betting environment the bookie does not bet, but serves as a go-between, enabling bettors to trade bets between themselves. Of course, in a free market with full information, the bettors do not need the bookie, but we assume here that bettors can only buy (and sell) bets from and to the bookie. For example, if one bettor believes that the probability of a certain event $A$ is $\frac{3}{4}$, while another bettor believes this probability to be $\frac{1}{4}$, the bookie will announce her willingness to sell and buy, for a nominal fee, bets of $\$ 1$ on $A$ that pay $\$ 2$ if $A$ happens. The first bettor will bet on $A$ and the second bettor will sell the bookie a bet on $A$. If $A$ happens, the second bettor pays the bookie $\$ 2$ that she in turn pays back to the first bettor, if $A$ does not happen no further payments are made. In all cases the bookie nets the betting fees. Note that these calculations were made regardless of the bookie beliefs.

But even if the bookie has to bet, it will be wrong to conclude her beliefs from her rates, since the rate-fixing bookie is acting as a monopolist. For example, if the bookie believes that the probability of the event $A$ is $\frac{1}{3}$, but bettors believe that the probability of this event is $\frac{1}{2}$, then her best strategy is to set the odds on $A$ at $\frac{1}{2}-\varepsilon$. The bettors will thus bet on $A$, and each dollar bet on $A$ equals, from the bookie's perspective, to the lottery $\left(1, \frac{2}{3} ; 1-\frac{1}{0.5-\varepsilon}, \frac{1}{3}\right)$. The expected value of this lottery is (almost) $\frac{1}{3}$ (see also Corollary 1 below for a formal presentation of these claims).

In this paper we point out that as a behavioral rule, the first interpretation suffers from a related flaw. It ignores the fact that the odds ratio posted by a bookie is merely a strategic decision in a game being played against the pool of bettors. What we need to do is examine the equilibria of the underlying betting game in order to draw conclusions about the equilibrium odds. Below we show how to construct environments in which this betting 
game has subgame perfect equilibrium with incoherent odds, even though all players possess additive probabilities.

It is true that these incoherent odds leave the bookie vulnerable to arbitrage - it's just that our particular collection of bettors does not find this arbitrage opportunity to be their most attractive collection of bets. If the bettors were only to concentrate on the sure gain, they would have to behave in a maximin fashion, behavior which most decision theorists would reject. Once the game theoretic nature of Dutch book interactions is recognized, all bets are off as to the kind of behavior we should expect to see.

Naturally, there are some unusual things about these environments. We do not assume that the actual odds are common knowledge, or even commonly held. Indeed a difference of opinion is necessary for our structure. The second thing that we need that is a bit unusual is that our bettors are not expected utility maximizers. This does not bother us, since there is plenty of evidence that many decision makers do violate the predictions of expected utility theory (see, for example, Kahneman and Tversky [17], MacCrimmon and Larsson [21], and Machina [23]). A bookie who is interested in choosing the best strategy while confronting other people has to take the behavior of her opponent as given. Even if she herself follows the rules of expected utility theory (as we assume below), it will be foolish for her to act as though everyone else also follows this theory, when they do not.

The claims of the paper should be understood as being practical and behavioral, and not as normative statements. Since there is a growing literature (both in psychology and economics) of models that use non additive representations of beliefs, we think that it is important to show that these models cannot be dismissed on the false grounds that "everyone who follows these models will go bankrupt." We relate our analysis to this literature, and to the literature on dynamic consistency and Dutch books, in Section 5 below.

\section{A Game Theoretic Analysis}

We consider the following simple situation. There are two disjoint events $A$ and $B$ which exhaust the set of possible states. The bookie, who is an expected utility maximizer, posts prices $a$ and $b$ for one dollar bets on $A$ and $B$ respectively. Bettors place bets after the prices are posted. A bettor may either buy or sell bets at the posted prices. For our purposes we assume 
that there are two bettors, and that the bookie knows their preferences (over uncertain prospects) and their beliefs (concerning the likelihood of the two events $A$ and $B) .{ }^{1}$ We impose the following budget constraint on the bettors. Each bettor has only one dollar and is not permitted to buy on credit nor is he allowed to sell a bet (buy a negative quantity) unless he proves that he possesses sufficient funds to pay off in the event he loses.

Let $x_{i}$ denote the amount that bettor $i$ bets on event $A$, and let $y_{i}$ denote the amount on $B$. A negative value indicates a sale. As usual, for any number $x, x^{+}$denotes $\max \{x, 0\}$ and $x^{-}$denotes $\max \{-x, 0\}$. Note that $x=x^{+}-x^{-}$. We can write the budget constraint for a bettor facing prices $a$ and $b$ as

$$
x^{+}+y^{+}+\frac{x^{-}}{a}+\frac{y^{-}}{b} \leqslant 1+x^{-}+y^{-} .
$$

Without loss of generality, we may restrict attention to prices satisfying $a+$ $b \leqslant 1$. For suppose $a+b>1$. Then set $a^{\prime}=1-b$ and $b^{\prime}=1-a$, so $a^{\prime}+b^{\prime} \leqslant 1$. For bettor $i$, set $y_{i}^{\prime}=-(1-a) x_{i} / a$, and $x_{i}^{\prime}=-(1-b) y_{i} / b$. These new bets yield the same monetary payoffs in each event as the bets $x_{i}$ and $y_{i}$. Furthermore, they satisfy the budget constraint $x^{\prime}+y^{\prime} \leqslant 1$. Formally then, the strategy set of the bookie is

$$
S=\{(a, b): a \geqslant 0, b \geqslant 0, a+b \leqslant 1\} .
$$

Once a bettor knows the rates $a$ and $b$, he decides how much to buy and sell on the two events $A$ and $B$. His strategy set $T$ is the collection of all betting schemes satisfying his budget constraint:

$$
\begin{aligned}
T=\left\{(\hat{x}, \hat{y}): S \rightarrow \mathbb{R}^{2} \mid\right. & \hat{x}^{+}(a, b)+\hat{y}^{+}(a, b)+\frac{\hat{x}^{-}(a, b)}{a}+\frac{\hat{y}^{-}(a, b)}{b} \\
& \left.\leqslant 1+\hat{x}^{-}(a, b)+\hat{y}^{-}(a, b) \text { for all }(a, b) \in S\right\} .
\end{aligned}
$$

The fact that $(\hat{x}, \hat{y})$ is a function from $S$ to $\mathbb{R}^{2}$ reflects the fact that the bettor decides how much to bet only after he learns the rates $a$ and $b$.

\footnotetext{
${ }^{1}$ Alternatively, we may assume that there is a single bettor, but that the bookie has some doubt about its identity. She believes that there is an even chance that this single bettor is one of the above two bettors. Since the bookie is assumed to maximize expected utility, her behavior in both environments will be the same (see Harsanyi [15]).
} 
To complete the description of the game we need to specify payoffs as a function of the strategies. Let $U\left(a, b, x_{1}, y_{1}, x_{2}, y_{2}\right)$ denote the bookie's payoff, and let $V_{i}\left(a, b, x_{1}, y_{1}, x_{2}, y_{2}\right)$ denote the payoff of bettor $i$. Since each bettor's payoff depends only on his own bets, for simplicity we will write $V_{i}\left(a, b, x_{i}, y_{i}\right)$. The bookie moves first, so the appropriate equilibrium concept is subgame perfect equilibrium.

Definition $1 A$ subgame perfect equilibrium, or equilibrium for brevity, of the two bettor game is a vector $\left(a, b, \hat{x}_{1}, \hat{y}_{1}, \hat{x}_{2}, \hat{y}_{2}\right)$ in $S \times T \times T$ satisfying:

1. Bettors maximize their payoff taking a and $b$ as given. That is, for each bettor $i$ and for all $(a, b)$ in $S$,

$$
\left.V_{i}\left(a, b, \hat{x}_{i}(a, b), \hat{y}_{i}(a, b)\right) \geqslant V_{i}(a, b, x, y)\right)
$$

for all $(x, y)$ satisfying $x^{+}+y^{+}+\frac{x^{-}}{a}+\frac{y^{-}}{b} \leqslant 1+x^{-}+y^{-}$.

2. The bookie maximizes her payoff taking $\hat{x}$ and $\hat{y}$ as given functions of $a$ and $b$. That is,

$$
\begin{aligned}
& U\left(a, b, \hat{x}_{1}(a, b), \hat{y}_{1}(a, b), \hat{x}_{2}(a, b), \hat{y}_{2}(a, b)\right) \geqslant \\
& U\left(a^{\prime}, b^{\prime}, \hat{x}_{1}\left(a^{\prime}, b^{\prime}\right), \hat{y}_{1}\left(a^{\prime}, b^{\prime}\right), \hat{x}_{2}\left(a^{\prime}, b^{\prime}\right), \hat{y}_{2}\left(a^{\prime}, b^{\prime}\right)\right)
\end{aligned}
$$

for all $\left(a^{\prime}, b^{\prime}\right) \in S$.

The outline of the game is therefore this:

- The bookie announces rates $a$ and $b$ at which she is willing to either buy or sell bets.

- Each bettor with a budget constraint of $\$ 1$ will buy and sell bets at the posted odds so as to maximize his utility.

- Now that the bookie knows the lottery she will face for each given pair of odds $a$ and $b$, she will choose $a$ and $b$ to maximize her utility.

For the remainder of our results, the players are assumed to evaluate lotteries using one of the following three functionals. We explain the choice of these functionals at the end of the section. 
Rank-Dependent Utility (Quiggin [27]. For properties of this model see Chew, Karni and Safra [6], Luce [19], and Wakker [34]). According to this theory, there is a non-decreasing utility function $u$ and a continuous probability transformation function $g:[0,1] \rightarrow[0,1]$, strictly increasing and satisfying $g(0)=0$ and $g(1)=1$, such that the value of a lottery with (subjective) distribution function $F$ is

$$
V(F)=\int u(x) d(g \circ F)(x) .
$$

For a random variable taking on only two values, $x<y$, with probabilities $q$ and $1-q$, the formula for the value reduces to

$$
V(x, q ; y, 1-q)=u(x) g(q)+u(y)[1-g(q)]
$$

Cumulative Prospect Theory This model was offered by Tversky and Kahneman [32] as a modification of their earlier prospect theory [17], which was claimed to violate monotonicity. (For an axiomatization of cumulative prospect theory, see Wakker and Tversky [35]). According to this theory, decision makers evaluate separately the positive part and the negative part of each lottery (positive and negative with respect to the status quo point). The value of each part is computed by taking the expected utility with respect to distortions of the distribution function. Different distortion functions may be used for the positive and the negative parts of the distribution. Obviously, this model is more general than the rank-dependent utility model, where the same distortion function is used for the whole distribution. For a random variable taking on only two values, $x<y$, with probabilities $q$ and $1-q$, the formula for the value reduces to

$$
V(x, q ; y, 1-q)=u(x) g(q)+u(y) h(1-q)
$$

If $x y>0$, then $h(1-q)=1-g(q)$, which is the functional form of eq. (2), but if $x y<0$ (that is, $x<0<y$ ), then $h(1-q$ ) may be different from $1-g(q)$.

Disappointment Aversion (Gul [12]. This theory is by itself a special case of Chew's [5] semi-weighted utility theory). According to this theory, the value of a lottery is given by

$$
V(F)=\frac{\gamma(\alpha)}{\alpha} \int_{x>C(F)} u(x) d F(x)+\frac{1-\gamma(\alpha)}{1-\alpha} \int_{x<C(F)} u(x) d F(x)
$$


where $\alpha$ is the probability that $F$ yields an outcome above its certainty equivalent $C(F)$, and $\gamma(\alpha)=\alpha /[1+(1-\alpha) \beta]$ for some number $\beta$.

According to disappointment aversion theory, the decision maker evaluates outcomes that are better than the certainty equivalent of a lottery by using an expected utility functional with a utility function $u$. He similarly evaluates outcomes that are worse than the certainty equivalent. Finally, the value of a lottery is a weighted sum of these two evaluations.

For a random variable taking on only two values, $x<y$, with probabilities $q$ and $1-q$, the formula for the value reduces to the functional of eq. (2), where $g(q)=(1-p)(1-\beta) /[1+\beta(1-p)]$.

What is common to these three functionals is that they are not smooth at $x=y$. Suppose, for example, that $q=\frac{1}{2}$, and differentiate the right-hand side of eq. (2) with respect to $x^{-}$at $x=y$ to obtain $u^{\prime}(x) g(q)$, while the derivative with respect to $y^{+}$at this point is $u^{\prime}(y)[1-g(q)]=u^{\prime}(x)[1-g(q)]$. If $g\left(\frac{1}{2}\right) \neq$ $\frac{1}{2}$, then these two derivatives differ, even though smooth preferences would have implied the same derivative. This observation follows from a general property, common to all these preferences, called first order risk aversion (Segal and Spivak [31]). The non differentiability around the certainty line plays a crucial role in our construction. (See also the Remark at the end of the next section).

In the sequel we use eq. (2). Since it is special case of eq. (3), we obtain that our analysis can be applied to all of the above models. ${ }^{2}$ Note that when both $u$ and $g$ are concave (convex), the functional exhibits risk aversion (seeking), see Chew, Karni, and Safra [6]. If $g$ is the identity function, then this functional form reduces to expected utility.

\section{The Bettors' Decision Problem}

Let $q$ and $1-q$ denote a bettor's subjective probability of $A$ and $B$ (for simplicity, we delete the index $i$ ). Given the prices $a$ and $b$ where $a+b \leqslant 1$, we may assume that $x, y \geqslant 0$, that is, the bettor does not sell bets on either $A$ or $B$. Suppose, for example, that $y<0$. then as before, the bettor is indifferent between selling $y$ on $B$ and betting $-(1-b) y / b$ on $A$ at the rate

\footnotetext{
${ }^{2}$ As will become evident below, examples can also be constructed for the functional form of eq. (3) where $h(1-q) \neq 1-g(q)$. See the discussion at the end of Section 3 below.
} 
$a^{\prime}=1-b$. Since $a \leqslant a^{\prime}$, the bettor cannot be worse off by betting only on A. It thus follows that the bettor's budget constraint is

$$
x+y \leqslant 1
$$

(See eq. (1)). Betting $x$ on $A$ and $y$ on $B$, the bettor will face the following uncertain prospect

$$
\left(\frac{x}{a}-x-y, q ; \frac{y}{b}-x-y, 1-q\right)
$$

Assuming the functional form of eq. (2), the bettor's payoffs are given in Table 1.

\begin{tabular}{|c|c|}
\hline & Bettor's Value \\
\hline$\frac{x}{a}<\frac{y}{b}$ & $g(q) u\left(\frac{x}{a}-x-y\right)+[1-g(q)] u\left(\frac{y}{b}-x-y\right)$ \\
\hline$\frac{x}{a}>\frac{y}{b}$ & {$[1-g(1-q)] u\left(\frac{x}{a}-x-y\right)+g(1-q) u\left(\frac{y}{b}-x-y\right)$} \\
\hline$\frac{x}{a}=\frac{y}{b}$ & $u\left(\frac{x}{a}-x-y\right)=u\left(\frac{y}{b}-x-y\right)$ \\
\hline
\end{tabular}

Table 1: Bettor's Payoffs from Purchases of Bets $x$ on $A$ and $y$ on $B$

The next lemma simplifies the analysis of the bettors' best response behavior.

Lemma 1 If $u$ is (weakly) convex and if $a+b \leqslant 1$, then a bettor's optimal response is to Plunge by betting everything on $A$ or on $B$, or else Hedge by betting so as to receive the same payoff in either event. The payoffs are given in Table 2 .

Proof Suppose that $x / a<y / b$. Then

$$
y \geqslant y(a+b)>b(x+y) \Longrightarrow \frac{y}{b}-x-y \geqslant 0
$$




\begin{tabular}{|l|c|}
\hline Action & Bettor's value \\
\hline Plunge on $A$ & $u(-1) g(1-q)+u\left(\frac{1-a}{a}\right)[1-g(1-q)]$ \\
\hline Plunge on $B$ & $u(-1) g(q)+u\left(\frac{1-b}{b}\right)[1-g(q)]$ \\
\hline Hedge & $u\left(\frac{1-a-b}{a+b}\right)$ \\
\hline
\end{tabular}

Table 2: Bettor's relevant payoffs.

Hence the bettor should increase the outcome $\frac{y}{b}-x-y$ to its highest possible level by setting $y=1-x$ (see the first case in Table 1 ). Since $u$ is convex, optimization leads to a corner solution, that is, either $y=1$ (and $x=0$ ), or $x / a=y / b$. Similarly, if $x / a>y / b$, then the bettor should set $x=1$, or fall back to $x / a=y / b$.

The bettor's optimal strategy depends on which of the three options in Table 2 yields the highest utility. We start with the case of linear utility, $u(x)=x$. Define the parameters

$$
\alpha=\frac{g(1-q)}{1-g(1-q)}
$$

and

$$
\beta=\frac{1-g(q)}{g(q)}
$$

Since $g$ is concave, $\alpha \geqslant \beta$. Simple calculations prove the next lemma.

Lemma 2 If a bettor's utility function $u$ is linear and his probability transformation function $g$ is concave, then his optimal strategies are:

- Plunge on $A$ whenever $b / a \geqslant \alpha$;

- Plunge on $B$ whenever $b / a \leqslant \beta$;

- Hedge whenever $\beta \leqslant b / a \leqslant \alpha$. 
These strategies are depicted in Figure 1.

Note that when $g$ is linear, that is, when the bettor is risk neutral, $\alpha=\beta$ and he will buy either on $A$ or on $B$, but not on both, unless $b / a=\alpha=\beta$, in which case he is indifferent between all three strategies.

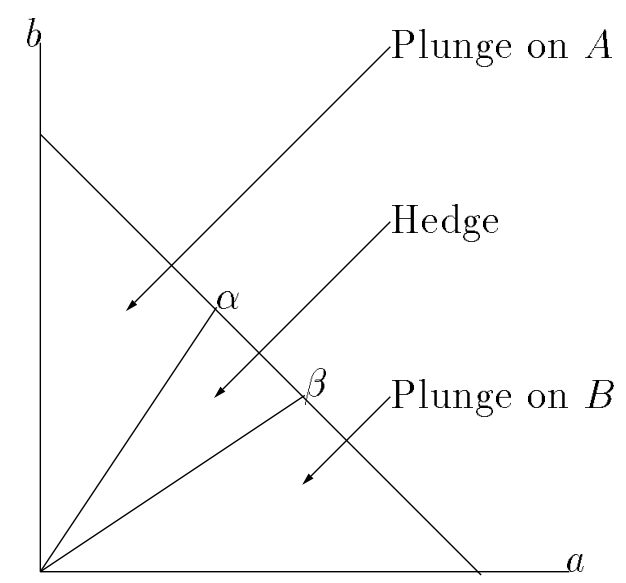

Figure 1: Bettor's optimal response (Linear utility)

It follows from Lemma 2 that if the bettor's utility function is linear, ${ }^{3}$ but his probability transformation function is concave, then his optimal strategy depends only on the ratio $b / a$. Since the bettor is buying bets, the bookie always prefers to raise both prices proportionately. We thus get the following result.

Theorem 1 If all bettors maximize the functional form of eq. (2) with linear utility functions and concave probability transformation functions, and the bookie's preferences are monotonic, then the bookie's equilibrium strategy satisfies $a+b=1$.

\footnotetext{
${ }^{3} \mathrm{~A}$ functional form satisfying this requirement was suggested by Yaari [36].
} 
In the special case of identical expected value maximizing bettors, the bookie will set the prices equal to the bettors' subjective probabilities, so that they are indifferent among all bets. Otherwise, the bettors will bet everything on the event whose price is less than its subjective probability, and the bookie benefits by raising the price of this event. In this case, the bookie's prices are the bettor's subjective probabilities, not her own.

Corollary 1 With only one type of bettor, if $u$ and $g$ are linear (i.e., the bettor is an expected value maximizer), then the equilibrium strategy of the bookie is to set $a=q$ and $b=1-q$ ( $q$ and $1-q$ are the bettor's subjective probability of $A$ and $B$ ).

In light of Theorem 1, if we want to create an equilibrium situation where $a+b<1$, it must be the case that not all utility functions are linear. From Table 2 we learn that the bettor:

- Prefers to plunge on $A$ than to plunge on $B$ iff

$$
\begin{gathered}
u(-1) g(1-q)+u\left(\frac{1-a}{a}\right)[1-g(1-q)] \geqslant \\
u(-1) g(q)+u\left(\frac{1-b}{b}\right)[1-g(q)]
\end{gathered}
$$

- Prefers to plunge on $A$ than to hedge iff

$$
u(-1) g(1-q)+u\left(\frac{1-a}{a}\right)[1-g(1-q)] \geqslant u\left(\frac{1-a-b}{a+b}\right)
$$

- Prefers to plunge on $B$ than to hedge iff

$$
u(-1) g(q)+u\left(\frac{1-b}{b}\right)[1-g(q)] \geqslant u\left(\frac{1-a-b}{a+b}\right)
$$

These equations determine the sets of $(a, b)$ pairs marking the bettor's indifference between each pair of strategies. For reasons that will become clear soon, we would like these border lines to be as in the left panel of Figure 2. In that case, the areas where each of the three strategies is optimal are as depicted on the right panel of this figure. The regions are labelled with 

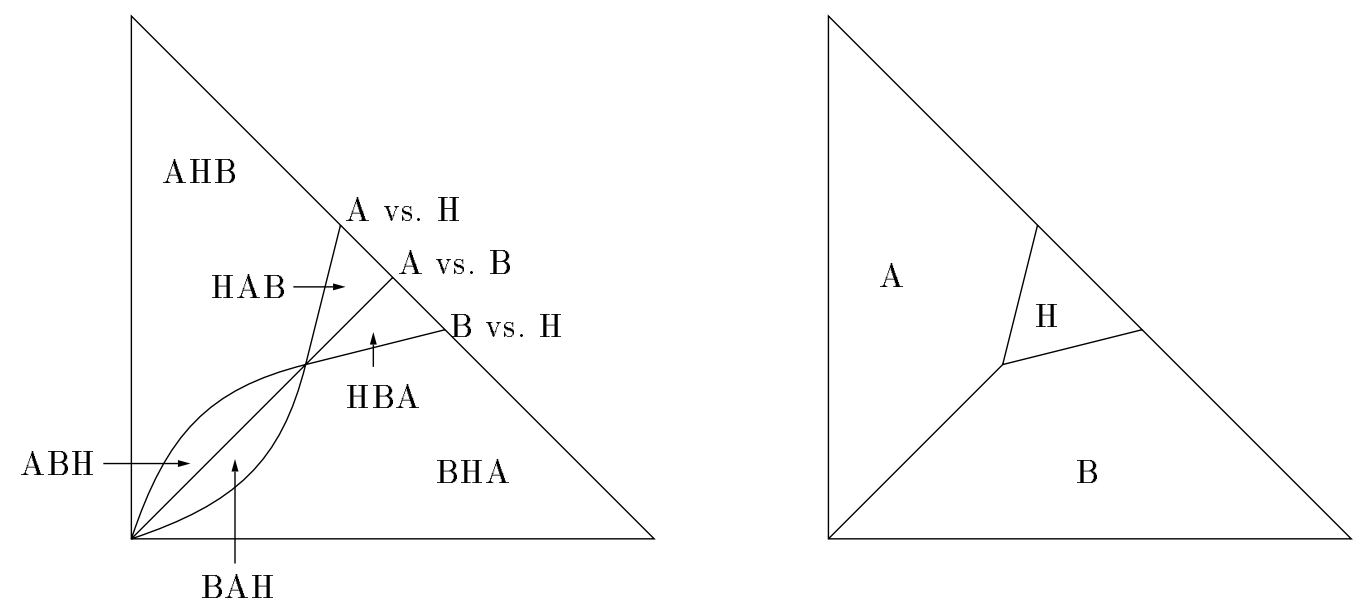

Figure 2: Bettor's choices

the bettor's preferences. That is, in the region marked "AHB," the bettor prefers plunging on $A$ to hedging to plunging on $B$.

It is not hard to come up with numerical examples that will lead to such a picture. Note that the transitivity of indifference guarantees that if two of these curves intersect, then all three of them intersect at the same point. Denote $\gamma=1-g(1-q)$ and $\delta=1-g(q)$, and assume wlg that $u(-1)=0$. Rewriting eq. (7)-(9), we obtain that the line of indifference $A$ vs. $B$ is given by

$$
b=\left[u^{-1}\left(\frac{\gamma}{\delta} u\left(\frac{1-a}{a}\right)\right)+1\right]^{-1}
$$

The line of indifference $\mathrm{A}$ vs. $\mathrm{H}$ is given by

$$
b=\left[u^{-1}\left(\gamma u\left(\frac{1-a}{a}\right)\right)+1\right]^{-1}-a
$$

And the line of indifference $\mathrm{B}$ vs. $\mathrm{H}$ is given by

$$
b=\left[u^{-1}\left(\delta u\left(\frac{1-b}{b}\right)\right)+1\right]^{-1}-b
$$


The following example will produce curves as in Figure 2. Let the utility $u$ for a bettor be of the following form.

$$
u(x)= \begin{cases}e^{k x} & x \geqslant 0 \\ x+1 & x \leqslant 0\end{cases}
$$

For $k \geqslant 1$ this is a (weakly) convex increasing function. Set

$$
s=-\ln [1-g(1-q)]
$$

and

$$
t=-\ln [1-g(q)]
$$

The parameters $s$ and $t$ depend only on the bettor's belief $q$ and his preferences through $g$. By choosing $q$ and the concave function $g$ carefully, we can choose $s$ and $t$ to be arbitrary positive numbers.

Eq. (10)-(12) can now be rewritten as

$$
\begin{gathered}
b=\frac{a k}{k-a(s-t)} \quad(\mathrm{A} \text { vs. B) } \\
b=\frac{a^{2} s}{k-a s} \quad(\mathrm{~A} \text { vs. H }) \\
a=\frac{b^{2} t}{k-b t} \quad(\mathrm{~B} \text { vs. H })
\end{gathered}
$$

Figure 2 depicts these loci for the case $s=1.8, t=1.5$, and $k=1$, although it is not drawn to scale.

Remark Although our analysis was done with respect to only three functional forms (rank-dependent utility, cumulative prospect theory, and disappointment aversion), it is clear how it can be extended to more general utility functions. In order to create areas as in Figure 2, we need to evaluate the lottery of eq. (6) by using a functional form such that for the set of bets satisfying $x+y=k$, the functional is convex in $x$, and has a kink at the point $x=a y / b$. The convexity is needed to guarantee that the bettor will either bet on $A$, or on $B$. The kink is needed to make sure that hedging may also be optimal. Such a kink is closely related to the concept of first order risk aversion (see Segal and Spivak [31]), a condition that is satisfied by all three functional forms discussed above. 


\section{The Bookie's Optimal Strategy}

Suppose that when the bettor is indifferent between plunging and hedging, the bettor will plunge. (This will turn out to be the case in our equilibrium.) Even in this case, if there is only one bettor, the logic that when the bettor is plunging, the bookie wants to raise the price of the bettor's bet and when he's hedging, the bookie wants to raise both prices, drives the equilibrium prices to satisfy $a+b=1$. Of course, since the boundary lines are nonlinear, the bookie may have to change the price ratio while raising the prices.

It is this phenomenon that allows us to construct an equilibrium with $a+b<1$. Since the set of directions in which we can raise prices depends on the prices, if there are heterogeneous bettors, these sets of directions may not overlap. That is, it may be impossible to raise prices and keep both bettors making the same bets.

Suppose that the bookie is maximizing an expected utility functional and that there are two bettors, I and II, with optimal strategies as indicated in Figure 3. There are five points of special interest, labelled $P, Q, R, S$, and $T$. Point $Q$ has the largest $a$ for which both bettors will plunge on $A$, and point $T$ has the largest $b$ for which both bettors will plunge on $B$. At point $R$, Bettor I hedges while II plunges on $A$. At $S$, I plunges on $B$, while II hedges. The segment joining $R$ and $S$ has both bettors hedging. Finally, at point $P$, Bettor I is plunging on $B$ and Bettor II is plunging on $A$. It is easy to see that the bookie's expected utility will be maximized at one of $P, Q, T$, or on the segment $\overline{R S}$. Letting $p$ and $1-p$ denote the bookie's subjective probabilities of $A$ and $B$, her expected utilities are given in Table 3 .

It is possible to choose values for $p, s_{I}, t_{I}, k_{I}, s_{I I}, t_{I I}$, and $k_{I I}$, and a concave increasing utility $u$ for the bookie so that point $P$ has the highest expected utility. For instance, choose $k_{I}=2.857, k_{I I}=1, s_{I}=28.57, t_{I}=1$, $s_{I I}=3, t_{I I}=12$ (Figure 3 is based on these values, although it is not drawn to scale) and $p=.2$. For the bookie's utility choose

$$
u(x)= \begin{cases}x & x \geqslant-2 \\ 4 x+6 & x \leqslant-2 .\end{cases}
$$

Then to three decimal places, the bookie's expected utilities are given in Table 4 . In the equilibrium of this game the bookie chooses $P$ and does not post additive prices. ${ }^{4}$

\footnotetext{
${ }^{4}$ The utility function given above is not differentiable at $x=-2$, but it can be smoothed
} 


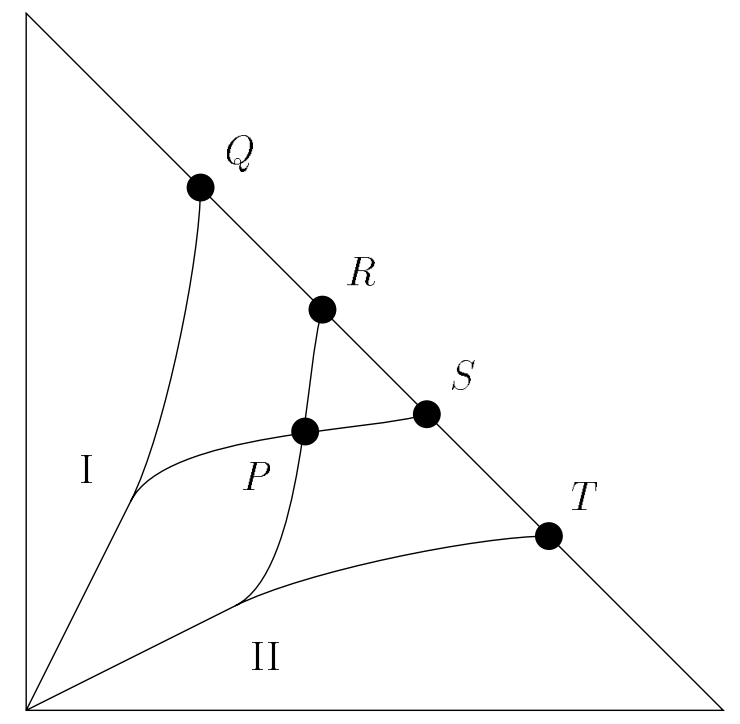

Figure 3: Two bettors

\begin{tabular}{|l|c|}
\hline$P$ & $p u\left(2-\frac{1}{a}\right)+(1-p) u\left(2-\frac{1}{b}\right)$ \\
\hline$Q$ & $p u\left(2-\frac{2}{a}\right)+(1-p) u(2)$ \\
\hline$R$ & $p u\left(1-\frac{1}{a}\right)+(1-p) u(1)$ \\
\hline$S$ & $p u(1)+(1-p) u\left(1-\frac{1}{b}\right)$ \\
\hline$T$ & $p u(2)+(1-p) u\left(2-\frac{2}{b}\right)$ \\
\hline$\overline{R S}$ & $u(0)$ \\
\hline
\end{tabular}

Table 3: Bookie's candidate strategies. 


\begin{tabular}{|l|c|c|}
\hline Point & $(a, b)$ & Expected utility \\
\hline$P$ & $(.248, .727)$ & 0.080 \\
\hline$Q$ & $(.091, .909)$ & -13.200 \\
\hline$R$ & $(.250, .750)$ & -0.400 \\
\hline$S$ & $(.259, .741)$ & -0.215 \\
\hline$T$ & $(.923, .077)$ & -71.600 \\
\hline$\overline{R S}$ & & 0.000 \\
\hline
\end{tabular}

Table 4: Bookie's candidate strategies: Numerical example.

To be fair, there is another equilibrium, where the bettors hedge when indifferent, in which the bookie posts additive prices and her expected utility is zero. Our point is that there is at least one equilibrium (in fact the bookie's favorite) in which she sets non additive prices.

\section{Conclusions}

In a related paper [4] we pointed out that strategic behavior on the part of the bookie may eliminate the Dutch book argument against violations of the law of conditional probability. The analysis there too involves two bettors (with different beliefs). A major difference between the results of the current paper and the results obtained in [4] is that here, at least in one equilibrium situation, the bookie's optimal strategy must involve a violation of probability theory. In [4], on the other hand, the most we can get is a situation where posting non-multiplicative rates is as good as using multiplicative ones.

Several recent nonexpected utility models are based on the assumption that decision makers do not obey some of the basic rules of probability theory. Schmeidler [30] and Gilboa [10] present models of behavior with non additive probabilities. One possible interpretation of the rank-dependent utility model (Quiggin [27]) is that it distorts the probabilities in such a way that

in a neighborhood of -2 without changing any of the relevant expected utilities. Thus we could specify differentiable utility with the same equilibrium. 
their values are no longer additive. This is clearly the meaning of the probability weighting function of Kahneman and Tversky's [17] prospect theory. It is this intuition that is carried over into their cumulative model [32]. We do not claim that the reason for these violations is that people behave strategically. Nor do we want to suggest that the correct interpretation of the above mentioned models is game theoretic. However, we believe that these models and empirical evidence cannot be rejected as irrelevant on the grounds that violations of probability theory expose the decision maker to a Dutch book. All of these models analyze the behavior of a single agent. Dutch books must involve at least two agents, therefore the correct framework is game theoretic, and one must assume that agents behave strategically. Traditional analyses of Dutch books assume that the person offering choices to the subject is much more sophisticated than the subject. Our approach is more symmetric in that the bookie is at least as sophisticated as the bettors.

Our major claim is that when people behave strategically, that is, when they are willing to deliberately misrepresent their beliefs and tastes in order to exploit some possible gains from trade, it is wrong to interpret the betting rates they announce as their subjective probabilities of the different events. Instead, these rates should be understood as the prices at which subjects are willing to trade certain goods (simple lotteries tickets). If the market is non-competitive and prices are manipulable by agents - and the framework of Dutch books is basically non-competitive-then the observed rates at which subjects are willing to exchange goods typically do not equal their true subjective rates (known in the economic literature as the marginal rate of substitution).

The idea that rules of behavior can be justified by Dutch books is not restricted to probability theory. In recent years there was a growing body of literature on the employment of Dutch books in enforcing dynamic consistency (see e.g. Green [11], Hammond [13, 14], Machina [24], Epstein and Le Breton [9], Border and Segal [3], and Sarin and Wakker [28]). These works either try to show that violations of dynamic consistency imply vulnerability to Dutch books (Green [11], Hammond [13, 14], and Border and Segal [3]), or they offer alternative definitions of dynamic consistency that do not expose decision makers to Dutch books. None of these models assumes strategic behavior on the side of the decision maker. The analysis of such a behavior may well change our view of dynamic consistency. 


\section{References}

[1] Abdellaoui, M. 2001. "A genuine rank-dependent generalization of the von Neumann-Morgenstern expected utility theorem," Econometrica, forthcoming.

[2] Allais, M. 1953. "Le comportement de l'homme rationnel devant le risque: Critique des postulats et axiomes de l'ecole Americaine," Econometrica $21: 503-546$.

[3] Border, K.C. and U. Segal. 1994. "Dynamic consistency implies approximately expected utility," Journal of Economic Theory 63:170-188.

[4] Border, K.C. and U. Segal. 1994. "Dutch books and conditional probability," Economic Journal 104:71-75.

[5] Chew, S.H. 1989. "Axiomatic utility theories with the betweenness property," Annals Op. Res. 19:273-298.

[6] Chew, S.H., E. Karni, and Z. Safra. 1987. "Risk aversion in the theory of expected utility with rank dependent preferences," Journal of Economic Theory 42:370-381.

[7] de Finetti, B. 1964. "Foresight: Its logical laws, its subjective sources." In H. E. Kyburg, Jr. and H. E. Smokler, eds., Studies in Subjective Probability, pp. 57-118. Wiley, New York.

[8] Edwards, W. 1955. "The prediction of decisions among bets," Journal of Experimental Psychology 50:201-214.

[9] Epstein, L.G. and M. Le Breton. 1994. "Dynamically consistent beliefs must be Bayesian," Journal of Economic Theory

[10] Gilboa, I. 1987. "Expected utility with purely subjective non-additive probabilities," Journal of Mathematical Economics 16:65-88.

[11] Green, J.R. 1987. “' 'Making book against oneself,' the independence axiom and non-linear utility theory," Quarterly Journal of Economics 102(4):785-796. 
[12] Gul, F. 1991. "A theory of disappointment aversion," Econometrica $59: 667-686$.

[13] Hammond, P. 1988: "Consequentialism and the independence axiom." In Risk, decision, and rationality. Ed.: B. Munier. Dordrecht, Holland: D. Reidel, pp. 503-515.

[14] Hammond, P. 1988: "Consequentialist foundations for expected utility," Theory and Decision 25:25-78.

[15] Harsanyi, J. 1967: "Games with incomplete information played by "Bayesian" players," Management Science 14:159-182.

[16] Heath, D. and W. Sudderth. 1972. "On a theorem of de Finetti, oddsmaking, and game theory," Annals of Mathematical Statistics 43:20722077 .

[17] Kahneman, D. and A. Tversky. 1979. "Prospect theory: An analysis of decision under risk," Econometrica 47:263-291.

[18] Lopes, L.L. and G.C. Oden. 1999. "The role of aspiration level in risky choice: A comparison of cumulative prospect theory and SP/A theory," Journal of Mathematical Psychology 43:286-313.

[19] Luce, R.D. 1988. "Rank dependent, subjective expected-utility representations," Journal of Risk and Uncertainty 1:305-332.

[20] Luce, R.D. 2001. "Reduction invariance and Prelec's weighting functions," Journal of Mathematical Psychology 45:167-179.

[21] MacCrimmon, K.R., and S. Larsson. 1979.: "Utility theory: Axioms versus "paradoxes'." In M. Allais and O. Hagen, Eds.: Expected Utility Hypotheses and the Allais Paradox. Dordrecht: D. Reidel.

[22] Machina, M.J. 1982. “'Expected utility' analysis without the independence axiom," Econometrica 50:277-323.

[23] Machina, M.J. 1987. "Choice under uncertainty: problems solved and unsolved," Journal of Economic Perspectives 1:121-154. 
[24] Machina, M.J. 1989. "Dynamic consistency and non-expected utility models of choice under uncertainty," Journal of Economic Literature $26: 1622-1668$.

[25] Miyamoto, J.M. 1999. “Quality-adjusted life years (QALY) utility models under expected utility and rank dependent utility assumptions," Journal of Mathematical Psychology 43:201-237.

[26] Prelec, D. 1998. "The probability weighting function," Econometrica $66: 497-528$.

[27] Quiggin, J. 1982. "A theory of anticipated utility," Journal of Economic Behavior and Organization 3:323-343.

[28] Sarin, R. and P.P. Wakker. 1993. "Consistency in Dynamic choice situations," mimeo.

[29] Savage, L.J. 1954. Foundations of Statistics, New York, John Wiley.

[30] Schmeidler, D. 1989. "Subjective probability and expected utility without additivity," Econometrica 57:571-587.

[31] Segal, U. and A. Spivak, 1990. "First order versus second order risk aversion," Journal of Economic Theory 51:111-125.

[32] Tversky, A. and D. Kahneman. 1992. "Advances in prospect theory: Cumulative representation of uncertainty," Journal of Risk and Uncertainty $5: 297-323$.

[33] von Neumann, J. and O. Morgenstern. 1944. Theory of Games and Economic Behavior, Princeton, Princeton University Press.

[34] Wakker, P.P. 1994. "Separating marginal utility and probabilistic risk aversion," Theory and Decision 36:1-44.

[35] Wakker, P.P. and A. Tversky. 1993. "An axiomatization of cumulative prospect theory," Journal of Risk and Uncertainty 7:147-176.

[36] Yaari, M.E. 1987. "The dual theory of choice under risk," Econometrica $55: 95-115$. 